\title{
Post-Operative Behavioural Assessment of Cats Undergoing Ovariohysterectomy
}

Vigneswari Murugesan $^{1 *}$, Kumaresan Arunachalam²,
Kathirvel Shanmugam ${ }^{1}$ and Mekala Palanivel

${ }^{1}$ Department of Veterinary Surgery and Radiology, ${ }^{3}$ Department of Veterinary Pharmacology and Toxicology, Veterinary College and Research Institute,

Tamil Nadu Veterinary and Animal Sciences University, Namakkal - 637 002, India

${ }^{2}$ Department of Veterinary Surgery and Radiology, Veterinary College and Research Institute, Tamil Nadu Veterinary and Animal Sciences University, Orathanadu, Thanjavur 614625, India

*Corresponding author

\section{A B S T R A C T}

\begin{tabular}{|c|}
\hline Keywords \\
\hline $\begin{array}{l}\text { Feline, Glasgow } \\
\text { Composite Measure } \\
\text { Pain Scale, } \\
\text { Ovariohysterectomy }\end{array}$ \\
\hline Article Info \\
\hline $\begin{array}{l}\text { Accepted: } \\
\text { 08 June } 2020 \\
\text { Available Online: } \\
10 \text { July } 2020\end{array}$ \\
\hline
\end{tabular}

\section{Introduction}

Pain management is one of the most important steps of the perioperative period and has direct impact on quality of recovery. Opioids play an important role in the clinical management of pain in cats. Ideally, analgesics should be administered before noxious stimulation (preventive analgesia) with the aim of preventing sensitization of the central nervous system, which could lead to the development of hyperalgesia (Bortaolami and Love, 2015). Post-operative acute pain has aroused great interest due to its potential 
risk of developing into chronic pain if not treated properly, which worsens the recovery and quality of life of the patient Pergolizzi et al., (2014). Ovariohysterectomy (OHE) is one of the most common surgeries performed in cats. Besides reproductive control, the surgical procedure is indicated in cases of pyometra, metritis, dystocia, mammary tumours and reproductive disorders Oliveira et al., (2014).

Surgical trauma is associated with activation of the sympathetic-adrenal axis, with consequent breakdown of homeostasis and increased recovery time. Thus, less invasive techniques are being incorporated into the veterinary practice to enable better surgical recovery Oliveira et al., (2014).

Cats are one of the least studied species of domestic animals regarding pain recognition and its treatment (Al-Gizawiy and Rude, 2004). The inability of animals to self-report their symptoms provide a major challenge for observers attempting to assess pain and difficulty in postoperative treatment can often be inappropriate. Thus, the development of more accurate methods of evaluation is crucial for optimal management of pain.

Physiological changes like tachypnea, tachycardia, hypertension, mydriasis and salivation can be used as indicator for pain assessment in cats (Robertson, 2008). The aim of this study was to assess the post-operative pain in cats undergoing ovariohysterectomy through the Glasgow Composite Measure Pain Scale (CMPS-Feline) and in turn assess the analgesic rescue.

\section{Materials and Methods}

This work involved the use of client-owned animals only and followed individual clinical patient care. Ethical Approval from a Committee was not therefore needed.
Informed Consent in a written form was obtained from the owner of all animals described in this work for the procedures undertaken. The study was conducted in twenty apparently healthy queen cats subjected for ovariohysterectomy at Veterinary Clinical Complex, Veterinary College and Research Institute, Namakkal with the mean \pm SD body weight of $2.75 \pm$ $0.85 \mathrm{~kg}$. Food was withheld for 12 hours and given free access to water prior to anaesthesia. The selected cats were randomly divided into two groups comprising of ten animals each. Group I received dexmedetomidine $(10 \mu \mathrm{g} / \mathrm{kg})$ and ketamine $(5 \mathrm{mg} / \mathrm{kg})$ with butorphanol $(0.2 \mathrm{mg} / \mathrm{kg})$ and group II received dexmedetomidine $(10 \mu \mathrm{g} / \mathrm{kg})$ and ketamine $(5 \mathrm{mg} / \mathrm{kg})$ with fentanyl $(5 \mu \mathrm{g} / \mathrm{kg})$. In both the groups, maintenance of anaesthesia was carried out with isoflurane.

Ovariohysterectomy was performed in all the animals through right flank approach as per standard surgical procedure with the above anaesthetic protocol. Physiological variables like heart rate, respiratory rate and rectal temperature were assessed before and after drug administration at every 5 minutes interval.

After surgery, pain scores were assessed through CMPS-Feline continuously for 24 hours at five different time interval: one hour after surgery, two hours after surgery, five hours after surgery, twelve hours after surgery and twenty four hours after surgery. This scale comprises seven descriptive levels, which are ordered categorically. The total score was calculated from the sum of the items 'scores', ranging from 0 (no pain) to 20 (maximum pain).

Analgesic rescue was performed at the time the score reached 5 point and above (out of a total possible score of 20). The analgesic rescue was performed according to the need 
of each animal by intramuscular administration of $2 \mathrm{mg} / \mathrm{kg}$ of tramadol. The anaesthetic protocol chosen aimed to provide adequate analgesia during and after surgery, but without interfering directly in the pain assessment by the selected techniques.

Statistical analyses were performed using SPSS 20.0 version. The mean and standard errors were calculated and compared between both the groups. Two-way ANOVA evaluated pain scores within groups and student t-test compared pain scores between groups.

\section{Results and Discussion}

Total average score of CMPS-Feline recorded in the study as described in the questionnaire was calculated and are presented in (Table 1). All the cats were assessed for behavioural changes from each category at different time interval for analgesic intervention level. At one and two hours interval animal showed signs of sleeping, relaxed, ignoring the painful area and lack of response on gentle palpation of a surgical wound and scored 3.17 and 3.17 out of 20 in group I and II, respectively (Figure 1). At five hours interval animal showed signs of hunched/arched back posture, anxiousness, crying, response to stroking, painful reaction to surgical wound palpation, flatten ears with pulled back whiskers and scored 6.0 and 6.17 out of 20 in group I and II, respectively (Figure 2). At twelve and twenty four hours interval animal exhibited normal behaviour after adequate pain management showing normal position of ears, response to stroking, relaxed and normal response to palpation of surgical wound and scored 1.5 and 1.67 in group I and II, respectively (Figure 3 ).

CMPS-Feline is a simple tool and its updated version was the first valid instrument to relate psychometric analysis of clinical acute pain in cats also using facial expressions Reid $e t$ al., (2017). Facial expressions of acute pain in cats were described

Holden $e t$ $a l$., (2014) and incorporated into the new version of the CMPS-Feline. It evaluates acute pain in seven behavioural categories: vocalization, activity/posture, attention to wound, caricature observation (ears and muzzle), response to manipulation/stroking, response to palpation of wound and general behaviour. Scores are attributed to each category and the maximum final score is twenty. Behaviour based pain score was found effective for assessing the analgesic intervention level at different time interval for adequate pain management.

In the present study, pain thresholds measured after each time interval, mean score was calculated, which showed fentanyl group had a higher pain than butorphanol group, indicating that thermal nociceptive threshold values of butorphanol on intramuscular administration increases up to 8 hours. All the animals were given an analgesic rescue at $5^{\text {th }}$ hour postoperatively.

Table.1 Total pain score for behavioural changes at different postoperative time interval Glasgow CMPS - F (5 point and above (out of a total possible score of 20)

\begin{tabular}{|c|c|c|c|c|c|c|}
\hline Parameter & Group & \multicolumn{5}{|c|}{ Total score at different time intervals } \\
\cline { 3 - 7 } & & $\mathbf{1}$ & $\mathbf{2}$ & $\mathbf{5}$ & $\mathbf{1 2}$ & $\mathbf{2 4}$ \\
\hline \multirow{2}{*}{$\begin{array}{c}\text { Behavioural } \\
\text { changes }\end{array}$} & I & 3.50 & 3.50 & 6.17 & 1.67 & 1.67 \\
\cline { 2 - 7 } & II & 3.17 & 3.17 & 6.00 & 1.50 & 1.50 \\
\cline { 2 - 7 } & III & 3.17 & 3.17 & 6.17 & 1.67 & 1.67 \\
\hline
\end{tabular}



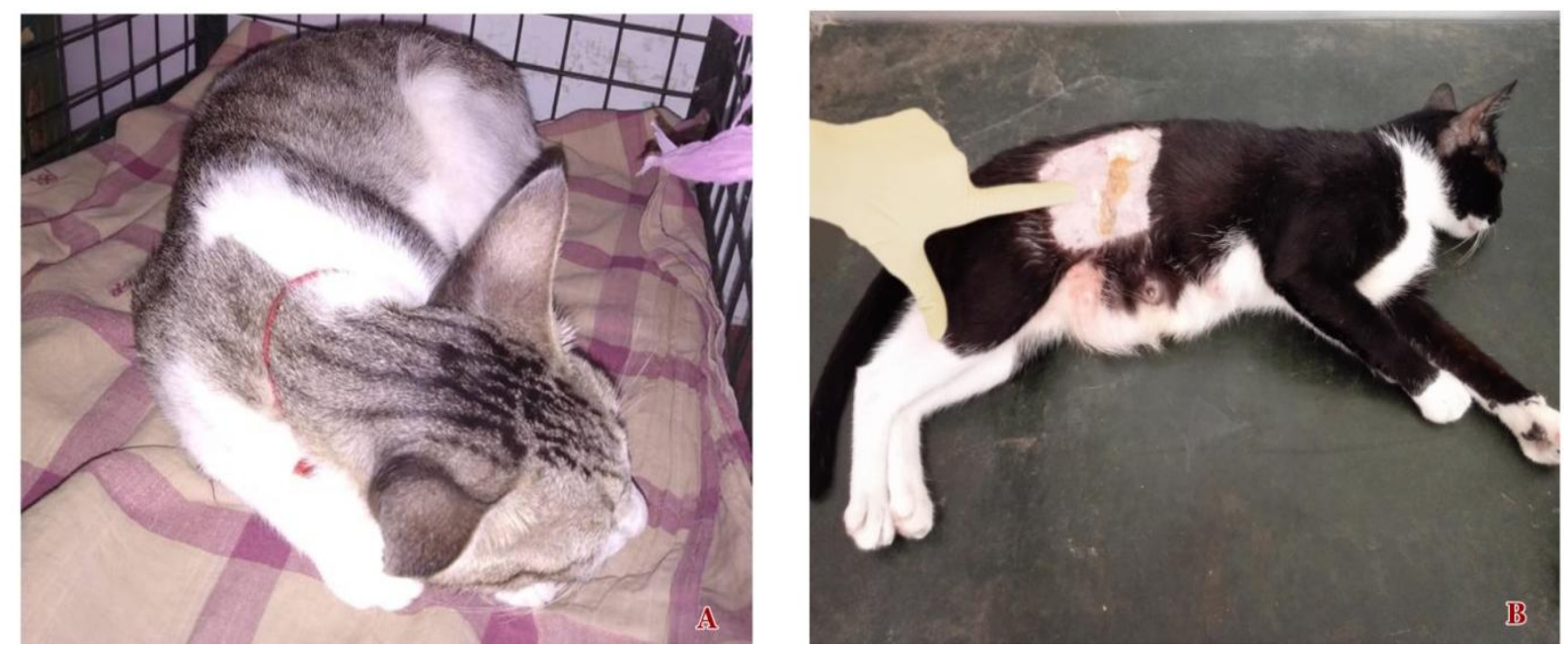

Fig.1 Behavioural changes at one hour postoperatively Glasgow CMPS - F

A) Sleeping / Relaxed

B) Lack of response on gentle palpation of a surgical wound
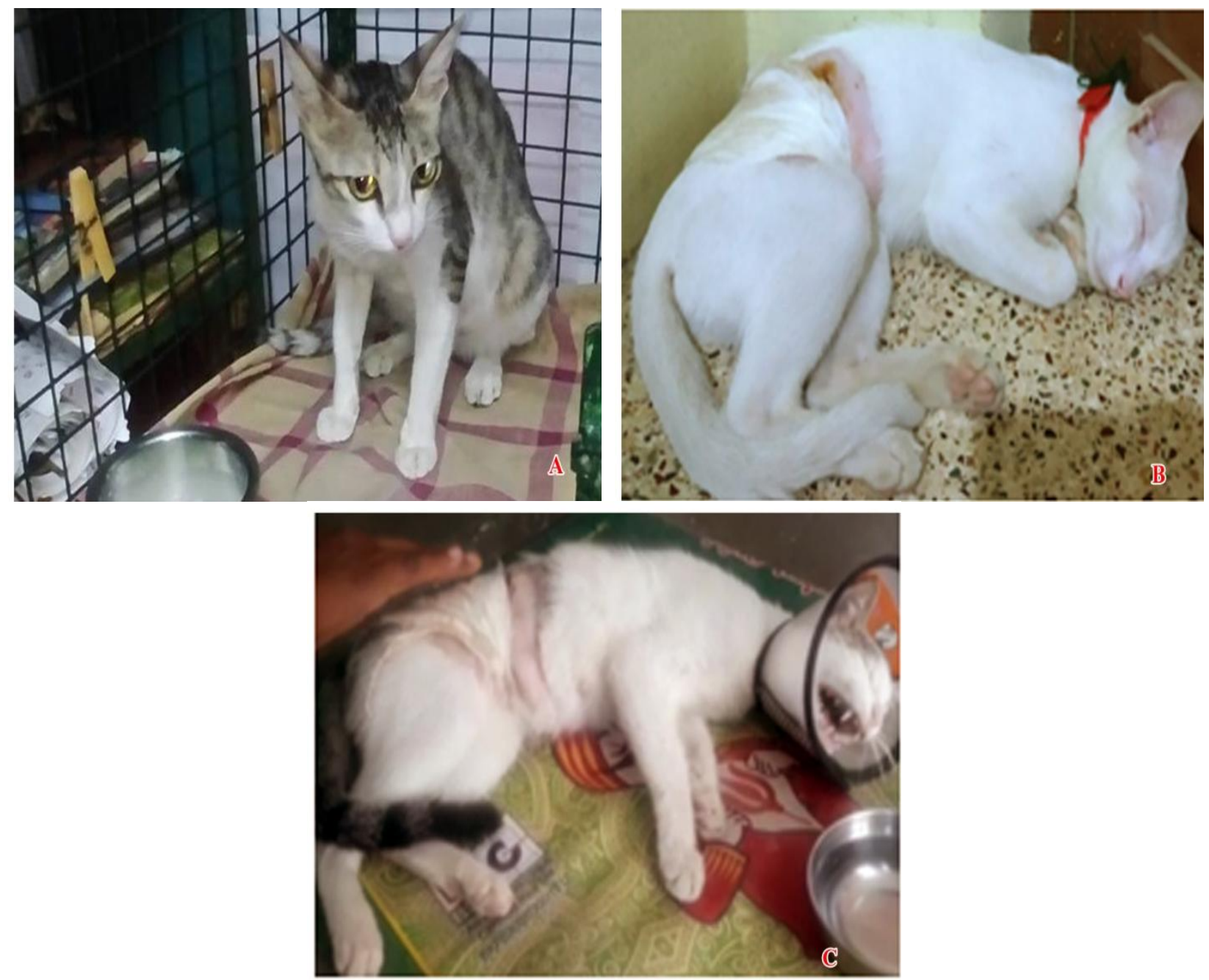

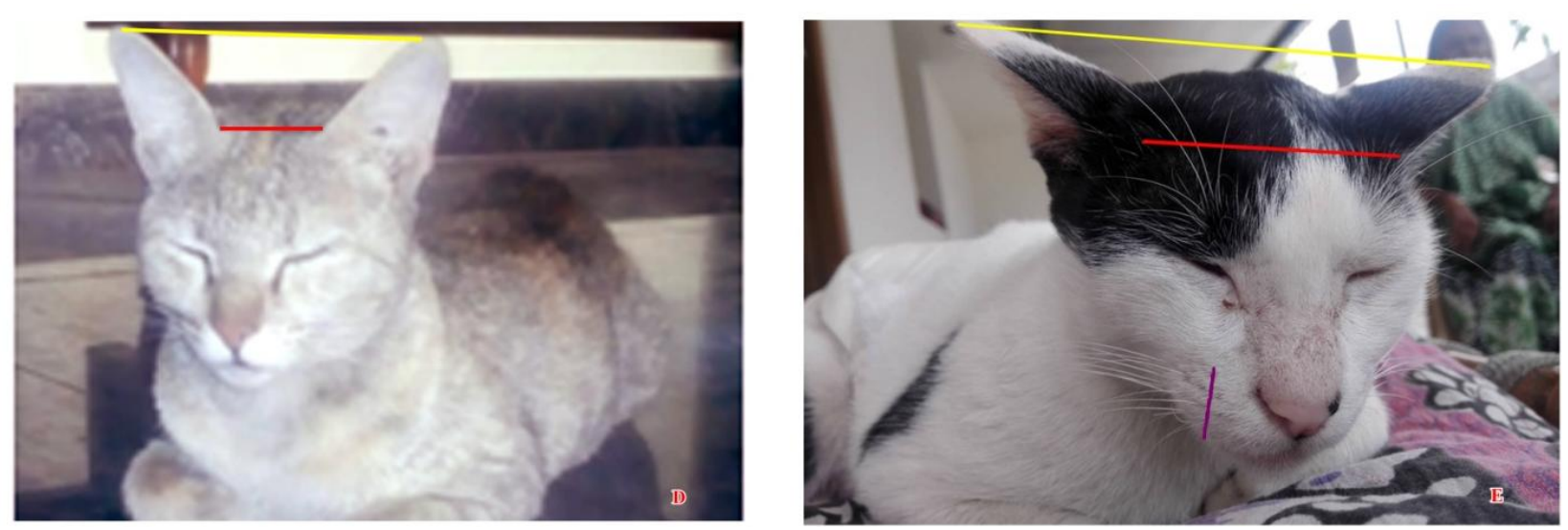

Fig.2 Behavioural changes at five hour postoperatively Glasgow CMPS - F

A) and B) Hunched posture and arched back indicative of pain

C) Facial expression indicative of painful reaction to surgical wound palpation

D) and E) Distance between the slope of the line joining the base of the ear and tip of the ear

Painful cat demonstrating squinted eyes, flatten ears and pulled back whiskers
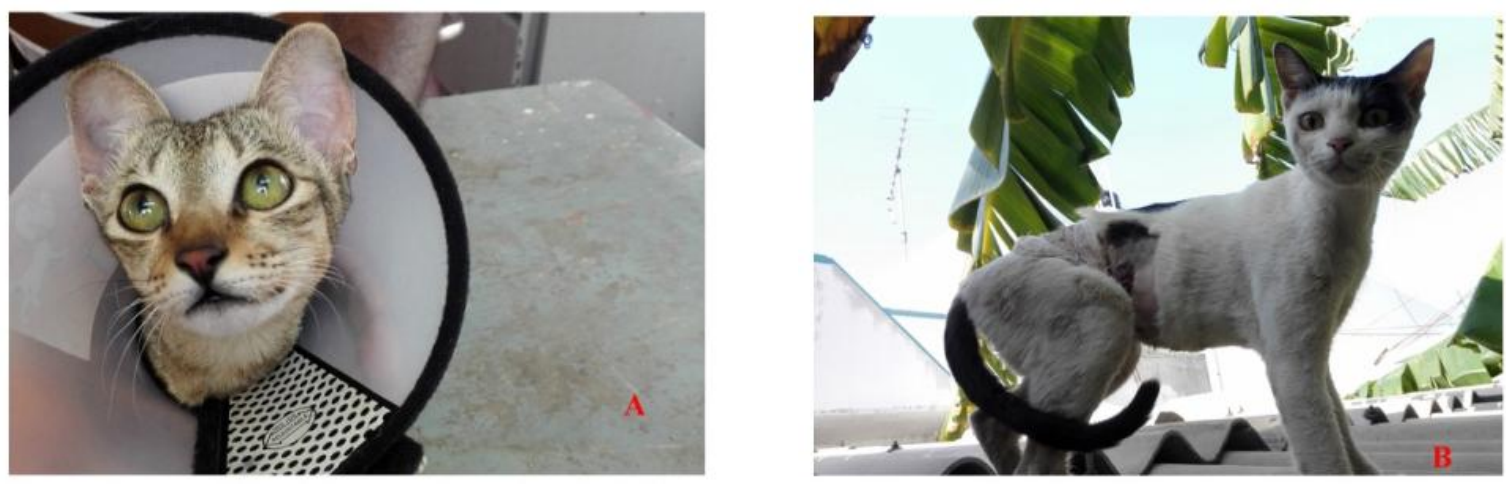

Fig 3: Behavioural changes at twenty fours hour postoperatively Glasgow CMPS - F

A) and B) Animal exhibiting normal behaviour after adequate pain management - normal position of ears and response to stroking

Postoperative behavioural changes observed at different time interval using CMPS Feline was found effective for assessing analgesic intervention level. The total pain score observed using CMPS Feline scale observed that fentanyl group had elevated pain score compared to butorphanol group indicating that butorphanol produced better postoperative analgesia compared to fentanyl.

\section{Acknowledgement}

The authors are thankful to the Tamil Nadu Veterinary and Animal Sciences University for the support during the study.

\section{References}

Al-Gizawiy, M.M and Rude, E.P., 2004. Comparison of preoperative carprofen and postoperative butorphanol as postsurgical analgesics in cats undergoing ovariohysterectomy. Veterinary Anaesthesia and Analgesia, 31: 164-174.

Bortaolami, E., and Love, E.J., 2015. Practical use of opioids in cats: a stateof-the-art, evidence-based review. Journal of Feline Medicine and Surgery, 17: 283-311

Brondani, J.T., Mama, K.R., Luna, S.P.L., 
Wright, B.D., Niyom, S., Ambrosio, J., Vogel, P.R., and Padovani, C.R., 2013. Validation of the English version of the UNESP- Botucatu multidimensional composite pain scale for assessing postoperative pain in cats. BMC Veterinary Research, 9: 143

Calvo, G., Holden, E., Reid, J., Scott, E.M., Firth, A., Bell, A., Robertson, S., and Nolan, A.M., 2014. Development of a behaviour - based measurement tool with defined intervention level for assessing acute pain in cats. Journal of Small Animal Practice, 55: 622-629

Holden, E., Calvo, G., Collins, M., Bell, A., Reid, J., Scott, E.M. and Nolan, A.M., 2014. Evaluation of facial expression in acute pain in cats. Journal of Small Animal Practice, 55: 615 - 621.

Oliveira, J. P., Mencalha, R., Sousa, C.A.S., Figueiredo, M.A., and Jorge, S.F.,
2014. Pain assessment in cats undergoing ovariohysterectomy by midline or lateral celiotomy through use of a previously validated multidimensional composite pain scale. Acta Cirúrgica Brasileira, 29: 635-636.

Pergolizzi junior, J.V., Raffa, R.B., and Taylor junior, R., 2014. Treating acute pain in light of the chronification of pain. Pain Management Nursing, 15: 380-390.

Reid, J., Scott, E.M., Calvo, G., and Nolan, A.M., 2017. Definitive Glasgow acute pain scale for cats: validation and intervention level. Veterinary Record, 180: 449.

Robertson, S. 2008. Managing pain in feline patients. Veterinary Clinics of North America: Small Animal Practice, 38: 1267-1290.

\section{How to cite this article:}

Vigneswari Murugesan, Kumaresan Arunachalam, Kathirvel Shanmugam and Mekala Palanivel. 2020. Post-Operative Behavioural Assessment of Cats Undergoing Ovariohysterectomy. Int.J.Curr.Microbiol.App.Sci. 9(07): 609-614. doi: https://doi.org/10.20546/ijcmas.2020.907.068 